\title{
Successful and long-term response to trastuzumab plus paclitaxel combination therapy in human epidermal growth factor receptor 2-positive extramammary Paget's disease: A case report and review of the literature
}

\author{
TAKASHI ICHIYAMA ${ }^{1,2}$, DAISUKE GOMI ${ }^{1}$, TOSHIROU FUKUSHIMA ${ }^{1}$, TAKASHI KOBAYASHI ${ }^{1}$, \\ NODOKA SEKIGUCHI ${ }^{1}$, AKIYUKI SAKAMOTO ${ }^{1}$, SHIGERU SASAKI ${ }^{1}$, KEIKO MAMIYA ${ }^{1}$, \\ TOMONOBU KOIZUMI ${ }^{1}$ and YOSHIHISA HAMA ${ }^{3}$ \\ ${ }^{1}$ Department of Comprehensive Cancer Therapy and ${ }^{2}$ First Department of Internal Medicine, \\ Shinshu University School of Medicine, Matsumoto, Nagano 390-8621; ${ }^{3}$ Department of \\ Surgery, Nagano Red Cross Hospital, Nagano, Nagano 380-0928, Japan
}

Received April 29, 2017; Accepted July 13, 2017

DOI: $10.3892 /$ mco.2017.1422

\begin{abstract}
A 58-year-old woman with a histologically confirmed diagnosis of vulvar extramammary Paget's disease (EMPD) was referred to our hospital due to locally advanced and relapsed EMPD. The patient had undergone surgical resection three times for relapsed vulvar EMPD over a period of 12 years, but developed locally advanced and unresectable EMPD. As pathological examination indicated that the lesion was positive for human epidermal growth factor receptor 2 (HER2) on immunohistochemical staining, the patient was treated with trastuzumab plus paclitaxel. The primary tumor mass and lymph node metastasis regressed successfully with combined trastuzumab and paclitaxel therapy, and the disease has been stable for $>2$ years after the initiation of treatment. These observations suggest that HER2 status must be determined in patients with advanced and/or metastatic extramammary Paget's disease and therapy with HER2 inhibitors should be considered as an option for the treatment of HER2-positive EMPD.
\end{abstract}

\section{Introduction}

Extramammary Paget's disease (EMPD) is a rare malignant skin tumor, which is considered to originate from cutaneous appendages and occurs in cutaneous areas bearing apocrine glands, such as the scrotum, vulva and axilla $(1,2)$. Generally,

Correspondence to: Professor Tomonobu Koizumi, Department of Comprehensive Cancer Therapy, Shinshu University School of Medicine, 3-1-1 Asahi, Matsumoto, Nagano 390-8621, Japan E-mail: tomonobu@shinshu-u.ac.jp

Key words: trastuzumab, paclitaxel, breast cancer, human epidermal growth factor receptor 2, chemotherapy the prognosis of EMPD is favorable if the primary lesion is completely resected and no metastases are present $(1,2)$. However, the prognosis of the progressive and metastatic disease is poor $(1,2)$, and data on the efficacy of cytotoxic chemotherapeutic agents in cases of advanced and/or metastatic EMPD are limited (3).

Several studies indicated that the tumor cells occasionally overexpress human epidermal growth factor receptor 2 (HER2) in patients with EMPD, at rates ranging from 15 to $65 \%$ (4-9). The humanized monoclonal antibody against HER2, trastuzumab, is expected to be effective in such cases. Indeed, several cases of advanced EMPD that exhibited a good response to trastuzumab, alone or combined with chemotherapy, were reported (10-14). However, there is currently insufficient evidence on the efficacy of treatment due to the rarity of the disease itself. We herein describe a case of HER2-positive and advanced vulvar EMPD, in which the primary tumor mass and lymph node metastasis regressed with combined trastuzumab and paclitaxel therapy for $>2$ years after initiation of treatment. The clinical course of this case is reported, along with a review of the relevant literature.

\section{Case report}

A 58-year-old woman was referred to the Shinshu University Hospital (Matsumoto, Japan) in August 2014 to receive further treatment for previously diagnosed EMPD. The findings of the physical examinations of the chest and abdomen were unremarkable, but a giant, slightly raised, erythematous and hemorrhagic granular lesion in the perineum and a mass on the inner thigh were identified (Fig. 1). Lower abdominal computed tomography (CT) revealed enlargement of the inguinal lesions, the mass in the perineum, and within the vagina (Fig. 2, arrows). The disease was initially diagnosed 12 years prior and the patient had undergone simple vulvectomy. The histological and immunohistochemical findings are presented in Fig. 3. However, the disease relapsed locally within the subsequent 4 years and the patient underwent vulvectomy twice and 


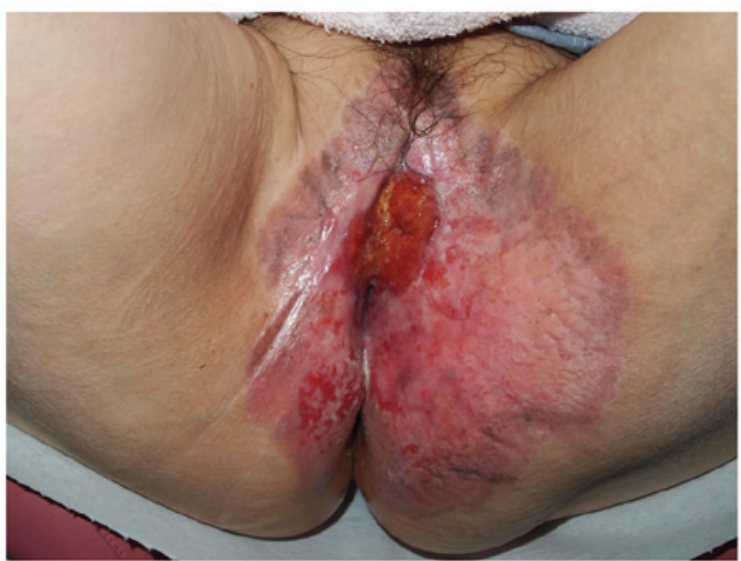

Figure 1. Giant, slightly raised erythematous and hemorrhagic granular lesions in the perineum and a mass in the inner thigh were observed on physical examination.

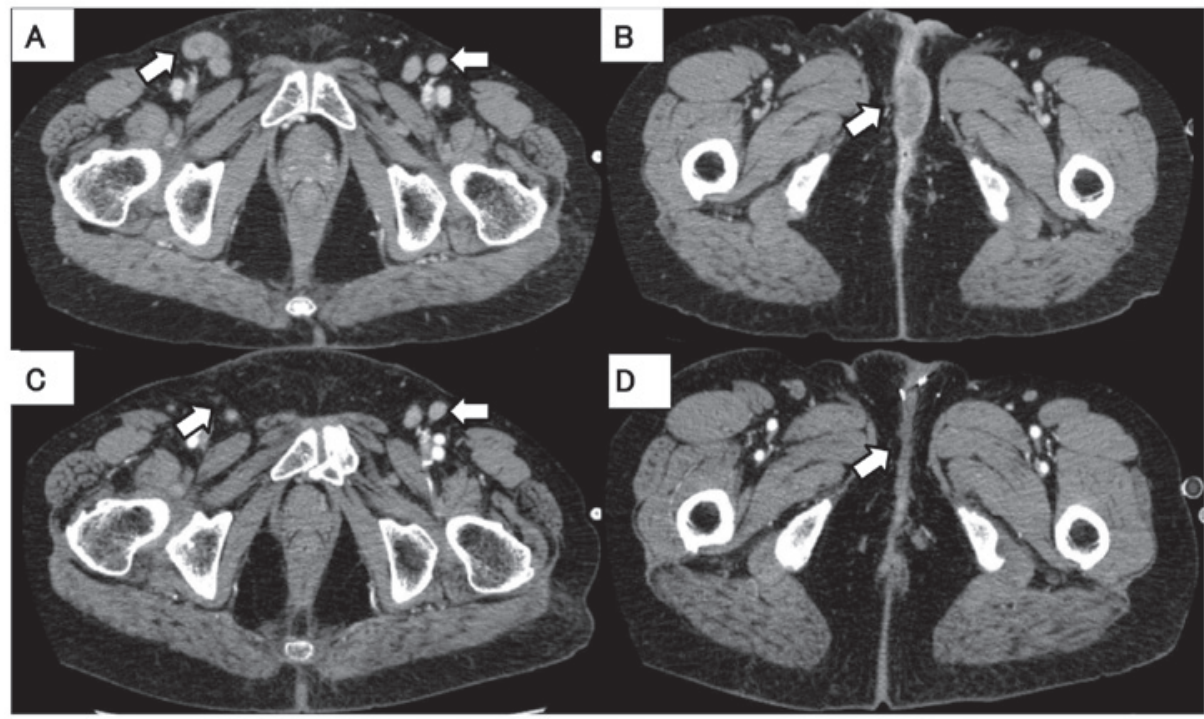

Figure 2. Changes in computed tomography (CT) findings (A and B) before and (C and D) after treatment with trastuzumab and paclitaxel. CT prior to therapy revealed (A) enlargement of the inguinal lymph nodes and (B) a mass in the perineum (arrows). (C and D) CT after therapy revealed disappearance of the inguinal lymph nodes and improvement of the mass in the perineum (arrows).
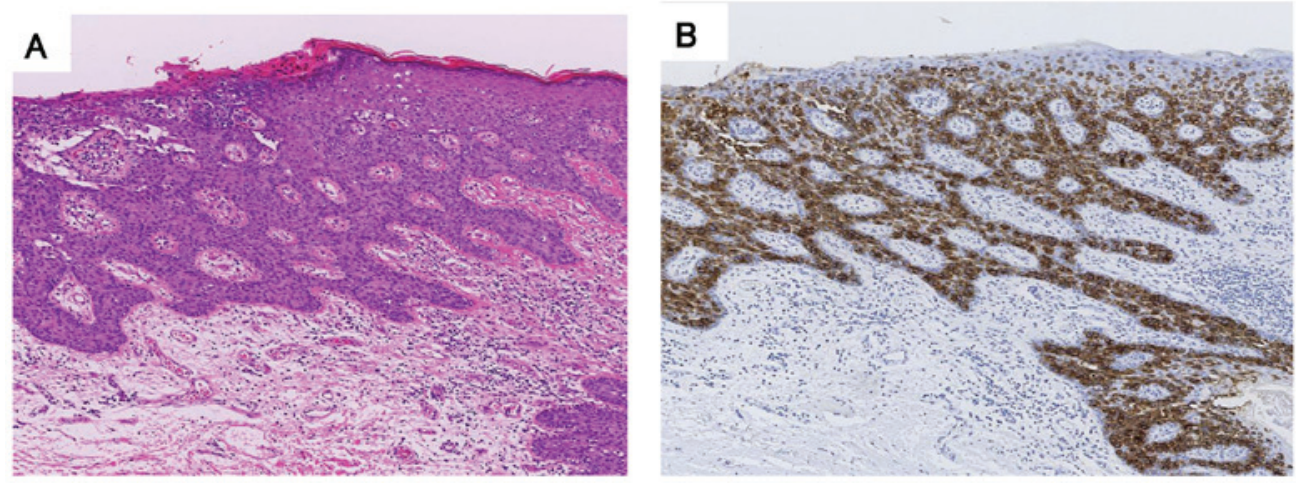

Figure 3. (A) Histological findings of vulvar excision specimens at initial presentation showed infiltration by hypertrophic cells with enlarged nuclei, proliferating as they replace the basal side of the epidermis (hematoxylin and eosin staining). (B) Immunohistological examination revealed that the tumor cells were positive for cytokeratin 7. All magnifications, $\mathrm{x} 40$.

cystostomy. In addition, episioplasty was performed for incontinence as a postoperative complication. Two years after the last operation, the disease relapsed with a tumor measuring $4 \mathrm{~cm}$. The patient refused further surgical treatment and was 


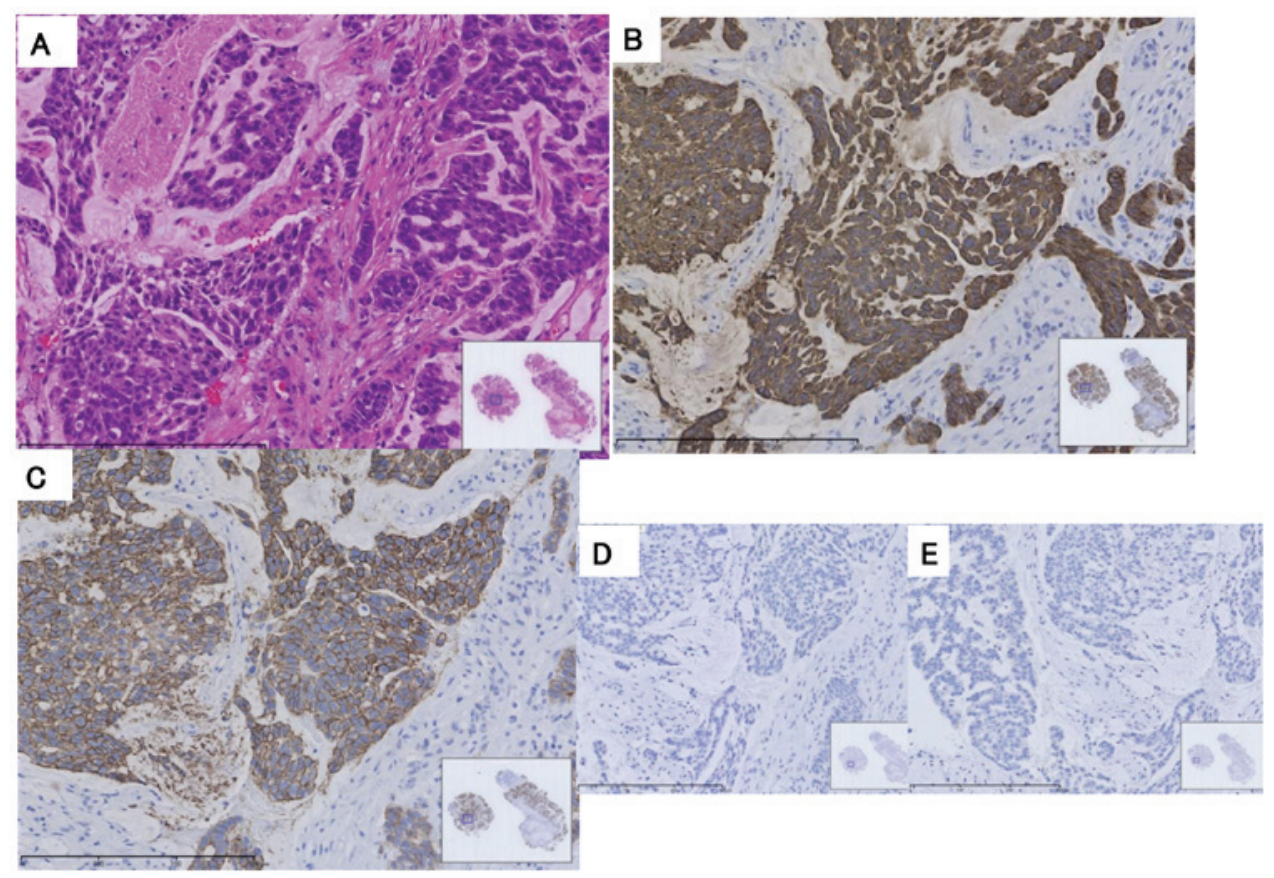

Figure 4. (A) Histological findings of biopsy specimens from the vulvar tumor revealed proliferation of hypertrophic cells with enlarged nuclei, with an irregular part on the basal side of the proliferation foci (hematoxylin and eosin staining). The immunohistochemical findings revealed the specimens to be positive for (B) cytokeratin 7 and (C) human epidermal growth factor 2, and negative for (D) estrogen and (E) progesterone receptors (all magnifications, x200).

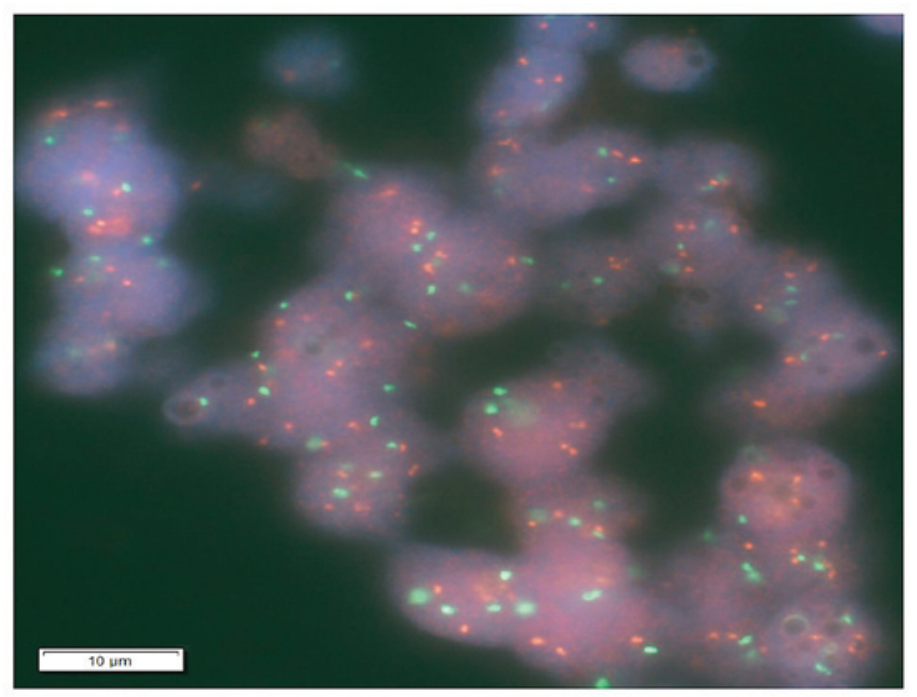

Figure 5. Human epidermal growth factor 2 (HER2) gene amplification by fluorescence in situ hybridization. Red signal, HER2 gene; green signal, chromosome 17 centromere (CEP17) (x600). The HER2 signal numbers and the signal numbers of CEP17 were counted and the HER2/CEP17 signal ratio was calculated as 2.1 , which was considered to be positive in the present case.

Table I. Previous cases of extramammary Paget's disease treated with trastuzumab reported in the English literature.

\begin{tabular}{|c|c|c|c|c|c|c|}
\hline Case & Age, years & Sex & Combination & $\begin{array}{c}\text { Duration of } \\
\text { observation, months }\end{array}$ & Others & (Refs.) \\
\hline 1 & 68 & Female & - & 12 & & (10) \\
\hline 2 & 71 & Male & - & 12 & Patient under hemodialysis & (11) \\
\hline 3 & 52 & Female & - & 12 & & (12) \\
\hline 4 & 70 & Female & Paclitaxel & 6 & & (13) \\
\hline 5 & 75 & Male & $\begin{array}{l}\text { Docetaxel followed } \\
\text { by paclitaxel }\end{array}$ & 6 & & (14) \\
\hline
\end{tabular}


followed up. Four years later, the tumors had increased in size and the patient experienced pain and difficulty walking; she was then referred to our hospital to receive chemotherapy or alternative therapy. The patient had a history of breast cancer prior to the development of EMPD, which was completely resected, and has been followed up without any signs of relapse. Biopsy of the vulval lesion was performed and the pathological findings confirmed EMPD (Fig. 4A). Immunohistochemical analysis revealed that tumor cells were positive for HER2 and cytokeratin (CK) 7, and were negative for estrogen and progesterone receptors (Fig. 4B-E).

The patient was treated with trastuzumab plus paclitaxel. Trastuzumab was administered at a loading dose of $4 \mathrm{mg} / \mathrm{kg}$ followed by $2 \mathrm{mg} / \mathrm{kg}$ weekly in combination with weekly paclitaxel at $80 \mathrm{mg} / \mathrm{m}^{2}$. No adverse effects, such as cardiotoxicity, neurotoxicity, or hematotoxicity, have been observed to date. The tumors decreased in size and the inguinal lymph nodes disappeared 6 months after the initiation of chemotherapy (Fig. 2C and D). This treatment has been continued for 2 years and 6 months, with partial response and good control of the clinical condition.

\section{Discussion}

We herein reported a case of HER2-positive advanced EMPD that responded successfully to trastuzumab and paclitaxel chemotherapy, achieving a prolonged disease-free period of $>2$ years after initiation of therapy. Kang et al (6) analyzed 246 Chinese male EMPD patients and reported that HER2-positive EMPD was observed at a rate of $65.1 \%$ based on immunohistological staining. Richter et al (7) also studied 39 patients with a histologically confirmed diagnosis of vulvar EMPD and reported that immunohistologically HER2-positive disease was observed in 58\% of the cases. Two Japanese studies reported HER2 overexpression in 16 of 104 cases $(15 \%)$ and 7 of 34 cases $(19.4 \%)(4,5)$. Thus, the frequency of HER2 overexpression in Japanese patients appeared to be lower compared with that in reports from other countries. However, HER 2 overexpression in these studies was correlated with biologically aggressive EMPD, i.e., local invasion and lymph node metastasis (4-9).

The patient presented herein had a history of resected breast cancer. The immunohistological findings were examined and the resected breast cancer was found to be negative for HER2 and CK7, suggesting that the EMPD was not a distant metastasis from the breast cancer and the biological profile was different between breast cancer and EMPD in the present case.

EMPD is a rare cutaneous malignant tumor, and analyses of HER2 overexpression or gene amplification in EMPD were performed in previously reported patients with surgically resected EMPD (4-9). Thus, there has been little clinical experience with advanced or metastatic EMPD. Indeed, there have been few reports regarding trastuzumab treatment in HER2-positive advanced or metastatic EMPD, with only five case reports identified in PubMed using the search terms 'EMPD' and 'trastuzumab' or 'HER2' (10-14). These previously reported cases are summarized in Table I. All the patients achieved a good response, but the observation time of treatment varied widely. Three cases involved trastuzumab monotherapy. It remains unclear whether the combination of cytotoxic drugs is beneficial for overall survival, progression-free survival, or response rate. Thus, further case studies of trastuzumab treatment for HER2-positive advanced EMPD are warranted, particularly focusing on the toxicities and/or time to progression.

In summary, we herein described a case of advanced EMPD with a long disease-free survival period of 2 years and 6 months after the initiation of combination therapy with trastuzumab and paclitaxel. Our observations suggest that HER2 expression should be determined in patients with advanced and/or metastatic EMPD, and therapy with HER2 inhibitors should be considered as an option for the treatment of HER2-positive EMPD.

The patient provided written informed consent regarding the publication of the case details and associated images.

\section{References}

1. Kyriazanos ID, Stamos NP, Miliadis L, Noussis G and Stoidis CN: Extra-mammary Paget's disease of the perianal region: A review of the literature emphasizing the operative management technique. Surg Oncol 20: e61-e71, 2011.

2. Parker LP, Parker JR, Bodurka-Bevers D, Deavers M, Bevers MW, Shen-Gunther J and Gershenson DM: Paget's disease of the vulva: Pathology, pattern of involvement, and prognosis. Gynecol Oncol 77: 183-189, 2000.

3. Tokuda Y, Arakura F and Uhara H: Combination chemotherapy of low-dose 5-fluorouracil and cisplatin for advanced extramammary Paget's disease. Int J Clin Oncol 20: 194-197, 2015.

4. Tanaka R, Sasajima Y, Tsuda H, Namikawa K, Tsutsumida A, Otsuka F and Yamazaki N: Human epidermal growth factor receptor 2 protein overexpression and gene amplification in extramammary Paget disease. Br J Dermatol 168: 1259-1266, 2013.

5. Ogawa T, Nagashima Y, Wada H, Akimoto K, Chiba Y, Nagatani T, Inayama Y, Yao M, Aoki I and Ikezawa Z: Extramammary Paget's disease: Analysis of growth signal pathway from the human epidermal growth factor receptor 2 protein. Hum Pathol 36: 1273-1280, 2005.

6. Kang Z, Zhang Q, Zhang Q, Li X, Hu T, Xu X, Wu Z, Zhang X, Wang $\mathrm{H}, \mathrm{Xu} \mathbf{J}$, et al: Clinical and pathological characteristics of extramammary Paget's disease: Report of 246 Chinese male patients. Int J Clin Exp Pathol 8: 13233-13240, 2015.

7. Richter CE, Hui P, Buza N, Silasi DA, Azodi M, Santin AD, Schwartz PE and Rutherford TJ: HER-2/NEU overexpression in vulvar Paget disease: The Yale experience. J Clin Pathol 63: 544-547, 2010 .

8. Keatings L, Sinclair J, Wright C, Corbett IP, Watchorn C, Hennessy C, Angus B, Lennard T and Horne CH: c-erbB-2 oncoprotein expression in mammary and extramammary Paget's disease: An immunohistochemical study. Histopathology 17: 243-247, 1990.

9. Plaza JA, Torres-Cabala C, Ivan D and Prieto VG: HER-2/neu expression in extramammary Paget disease: A clinicopathologic and immunohistochemistry study of 47 cases with and without underlying malignancy. J Cutan Pathol 36: 729-733, 2009.

10. Wakabayashi S, Togawa Y, Yoneyama K, Suehiro K, Kambe N and Matsue H: Dramatic cinical response of relapsed metastatic extramammary Paget's disease to trastuzumab monotherapy. Case Rep Dermatol Med 2012: 401362, 2012.

11. Barth P, Dulaimi Al-Saleem E, Edwards KW, Millis SZ, Wong YN and Geynisman DM: Metastatic extramammary Paget's disease of scrotum responds completely to single agent trastuzumab in a hemodialysis patient: Case report, molecular profiling and brief review of the literature. Case Rep Oncol Med 2015: 895151, 2015.

12. Karam A, Berek JS, Stenson A, Rao J and Dorigo O: HER-2/neu targeting for recurrent vulvar Paget's disease A case report and literature review. Gynecol Oncol 111: 568-571, 2008.

13. Hanawa F, Inozume T, Harada K, Kawamura T, Shibagaki N and Shimada S: A case of metastatic extramammary Paget's disease responding to trastuzumab plus paclitaxel combination therapy. Case Rep Dermatol 3: 223-227, 2011.

14. Takahagi S, Noda H, Kamegashira A, Madokoro N, Hori I, Shindo H, Mihara S and Hide M: Metastatic extramammary Paget's disease treated with paclitaxel and trastuzumab combination chemotherapy. J Dermatol 36: 457-461, 2009. 